\title{
Thrombotic Microangiopathy in IgA Nephropathy
}

\author{
Hamid Nasri ${ }^{1, *}$ \\ ${ }^{1}$ Department of Nephrology, Division of Nephropathology, Isfahan University of Medical Sciences, Isfahan, IR Iran \\ ${ }^{*}$ Corresponding author: Hamid Nasri, Department of Nephrology, Division of Nephropathology, Isfahan University of Medical Sciences, Isfahan, IR Iran. Tel: +98-3112208081, Fax: \\ +98-3112235043, E-mail: hamidnasri@med.mui.ac.ir
}

Received: January 11, 2013; Revised: January 14, 2013; Accepted: May 15, 2013

Keywords: Thrombosis; Thrombotic Microangiopathies; Nephropathy

\section{Dear Editor,}

Thrombotic microangiopathy (TMA) occurs in IgA nephropathy, but is uncommon in the setting of IgA nephropathy. Recently an article published by El Karoui and colleagues, entitled "A clinicopathologic study of thrombotic microangiopathy in IgA nephropathy" (1). They retrospectively examined a series of 128 patients diagnosed with IgA nephropathy (IgAN) between 2002 and 2008 who had a mean follow-up of $44 \pm 27$ months. They found that, $53 \%$ presented with lesions of thrombotic microangiopathy (TMA), acute or organized, in arteries and / or arterioles. They concluded that, lesions of TMA are frequent in IgA nephropathy and may occur in normotensive patients with near-normal renal histology (1). However, we would like to remind a few points about TMA in IgA nephropathy. In a study on 102 primary IgA nephropathy patients, we found, morphologic lesions of TMA in $2 \%$ of our patients, while all of them had malignant hypertension (2). In our study $71.6 \%$ of patients were male. The mean age of the patients was 37.7 years. Morphologic variables of MEST classification was as follows; M1: 90.2 \%, E: 32 \%, S: 67 \% also in grads I and II were in $30 \%$ and $19 \%$ respectively, while $51 \%$ were in grade zero. Previously in a study on a small group of IgAN patients with thrombotic microangiopathy injury, Chang et al. (3) found, association of TMA with advanced stages and severe proteinuria of IgA nephropathy. Few publications existed regarding the TMA in IgA nephropathy. However, it is uncommon in the setting of IgA nephropathy and its significance, as a concomitant histologic finding, is unclear $(4,5)$. It is possible that IgAN has different presentation between different regions (6-8). However, the reports of overlapping morphologic lesions of TMA and IgAN are poorly understood and debatable and needs more atten- tion in larger series of IgAN (8-10).

\section{Acknowledgements}

There is no acknowledgment.

\section{Authors' Contribution}

The article prepared by author.

\section{References}

1. El Karoui K, Hill GS, Karras A, Jacquot C, Moulonguet L, Kourilsky $\mathrm{O}$, et al. A clinicopathologic study of thrombotic microangiopathy in IgA nephropathy. JAm Soc Nephrol. 2012;23(1):137-48.

2. Nasri H, Mortazavi M, Ghorbani A, Shahbazian H, Kheiri S, Baradaran A, et al. Oxford-MEST classification in IgA nephropathy patients: A report from Iran. J Nephropathol. 2012;1(1):31-42.

3. Chang A, Kowalewska J, Smith KD, Nicosia RF, Alpers CE. A clinicopathologic study of thrombotic microangiopathy in the setting of IgA nephropathy. Clin Nephrol.2006;66(6):397-404.

4. Mubarak M. Oxford classification of IgA nephropathy: Broadening the scope of the classification. J Nephropathol. 2012;1(1):13-16.

5. Pipili C, Pantelias K, Papaioannou N, Paraskevakou H, Grapsa E. Hemolytic-uremic syndrome, malignant hypertension and IgA nephropathy: successful treatment with plasma exchange therapy. Transfus Apher Sci. 2012;47(2):155-8.

6. Ibrahim Seif E, Abdel-Salam Ibrahim E, Galal Elhefnawy N, Ibrahim Salman M. Histological patterns of idiopathic steroid resistant nephrotic syndrome in Egyptian children: A single centre study. J Nephropathol. 2013;2(1):53-60.

7. Shakeel S, Mubarak M, Kazi IJ, Jafry N, Ahmed E. Frequency and clinicopathological characteristics of variants of primary focal segmental glomerulosclerosis in adults presenting with nephrotic syndrome. J Nephropathol. 2013;2(1):28-35.

8. Assadi F. The epidemic of pediatric chronic kidney disease: the danger of skepticism. J Nephropathol. 2012;1(2):61-64.

9. Kari J. Epidemiology of chronic kidney disease in children. J Nephropathol. 2012;1(3):162-163.

10. Gheissari A, Hemmatzadeh S, Merrikhi A, Fadaei Tehrani S, Madihi Y. Chronic kidney disease in children: A report from a tertiary care center over 11 years. J Nephropathol. 2012;1(3):177-182.

Implication for health policy/practice/research/medical education:

Thrombotic microangiopathy (TMA) occurs in IgA nephropathy, but is uncommon in the setting of IgA nephropathy. However, the reports of overlapping morphologic lesions of TMA and IgAN are poorly understood and debatable and needs more attention in larger series of IgAN.

Copyright (c) 2013, Iranian Red Crescent Medical Journal; Published by Kowsar Corp. This is an open-access article distributed under the terms of the Creative Commons Attribution License, which permits unrestricted use, distribution, and reproduction in any medium, provided the original work is properly cited. 